\title{
Rhombencephalitis possibly caused by Mycoplasma pneumoniae
}

\author{
Paulo Pereira Christo ${ }^{1}$, Júlio Sérgio P. da Silva², \\ Ilana Vaula Werneck², Sandra Lucia Dias ${ }^{2}$
}

Mycoplasma pneumoniae is a frequent cause of respiratory infection in humans, especially schoolchildren, adolescents and young adults, accounting for about $20 \%$ of cases of pneumonia in this age group ${ }^{1}$. However, little is known about its extrapulmonary manifestations, with the central nervous system (CNS) being the most common extrapulmonary site which can be affected during any phase of the disease $^{2}$. About 1 to $10 \%$ of patients with pneumonia caused by $M$. pneumoniae require hospitalization due to some neurological complication related to this infection, and M. pneumoniae is possibly one of the leading causes of encephalitis in older children and adolescents ${ }^{2,3}$.

We report here the case of an adolescent girl with an uncommon manifestation of neurological complications characterized by the involvement of the brainstem and cerebellum likely caused by the M. pneumoniae in the absence of atypical pneumonia.

\section{CASE}

A 16-year-old girl, a student, born and living in Minas Gerais state, presented chest and neck pain, low fever, myalgia and coryza. Twenty-four hours after the onset of symptoms, the patient was seen at a basic health clinic and was first treated with common painkillers for viral symptoms, possibly upper airways viral infection. Forty-eight hours later, the patient presented transitory exanthema, headache, few episodes of vomiting and hyporexia, and continued to be febrile. Reassessment at a second health clinic showed dysarthria, gait ataxia and mental confusion, in addition to an episode of generalized tonic-clon- ic seizures. A cranial computed tomography performed 5 days later was normal. There was no report of important personal or family histories and no history of recent travel.

Seven days after the onset of symptoms, the patient was transferred to a tertiary care unit where the diagnostic hypothesis of HSV-1 meningoencephalitis was raised. At admission, the patient presented a preserved general state and was hydrated, acyanotic and eupneic. Examination of the respiratory and cardiologic system showed no abnormalities. Neurological examination showed the patient alert and oriented with isochoric and photoreactive pupils, neck stiffness, unaltered cranial nerves, dysmetria on the left side of the body, ataxic gait, normoactive osteotendinous reflexes, and the presence of a bilateral Babinski sign. Muscle strength and sensitivity were normal.

As part of the investigation, cerebrospinal fluid (CSF) examination showed the following results: leukocytes, 230 cells/ $\mathrm{mm}^{3}$ (90\% lymphocytes); red blood cells, 105 cells $/ \mathrm{mm}^{3}$; protein: $98 \mathrm{mg} / \mathrm{dL}$; glucose: $57 \mathrm{mg} / \mathrm{dL}$; nonreactive VDRL, negative acid-fast bacilli and China ink; negative cultures for bacteria, fungi and acidfast bacilli. PCR for herpes simplex virus type 1 and 2 was negative. Serological reactions showed the following results: dengue IgG and IgM negative; cytomegalovirus IgG positive/IgM negative; mononucleosis negative; Mycoplasma IgM positive at a titer of 1/512 and IgG negative. No immunological reactions for Mycoplasma were performed in CSF. Inflammatory tests showed P-ANCA and C-ANCA negative; ANF positive (1/80, punctiform pat-
Av. Alfredo Balena 189 / 1708 30130-100 Belo Horizonte MG - Brasil E-mail: ppc@gold.com.br

Received 1 June 2009

Received in final form 15 September 2009 Accepted 25 September 2009
TROMBOENCEFALITE PROVAVELMENTE CAUSADA PELO MYCOPLASMA PNEUMONIAE

Santa Casa de Belo Horizonte, Belo Horizonte MG, Brazil: 'MD, PhD, Department of Neurology.: ${ }^{2} \mathrm{MD}$, Department of Neurology. 

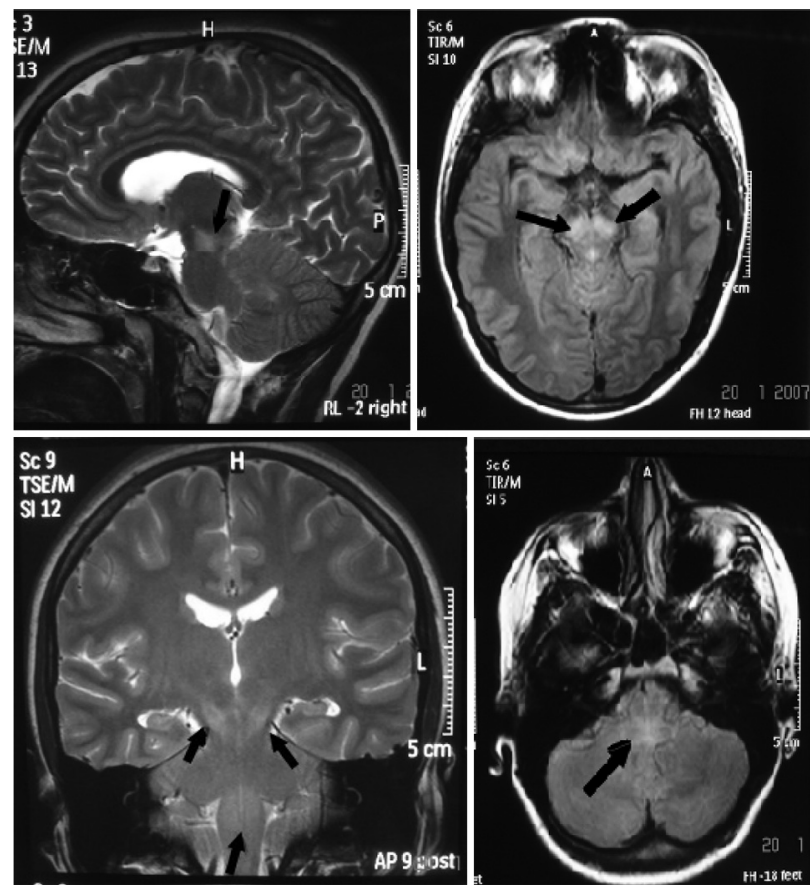

Figure. MRI of the brain shows abnormal signal intensity, hypointense lesions, at bilateral midbrain and cerebellum on T2-weighted and Flair images.

tern). Blood count, ions, CK and renal and hepatic function were normal. A chest X-ray showed no alterations. Magnetic resonance imaging (MRI) of the brain revealed hypointense lesions in the brainstem and cerebellum on T2-weighted and Flair images (Figure). Ten days after the first exams, the Mycoplasma IgM was negative and the IgG continued positive.

In view of the initial diagnostic suspicion of HSV-1 encephalitis, the patient received empirical treatment with Acyclovir for 10 days. Analysis of a new CSF sample on the seventh day of hospitalization revealed a reduction in cellularity and protein level. During hospitalization, the patient presented complete improvement of neurological symptoms and was discharged on day 14 after admission. Brain MRI performed 2 weeks after discharge showed improvement of radiological lesions and neurological reassessment after 6 months was normal.

The hospital ethic commission approved this case report and the parents gave informed consent for publication.

\section{DISCUSSION}

Pulmonary infection with $M$. pneumoniae can be asymptomatic and, in most cases, respiratory infection precedes or occurs concomitantly with CNS injury ${ }^{3}$. The interval between the onset of respiratory symptoms and neurological manifestations ranges from 2 to 14 days, and no respiratory infection is detected in $18 \%$ of cases ${ }^{2}$.

The most frequent extrapulmonary sites of M. pneu- moniae infection include the CNS, heart, skin, musculoskeletal and hematological system, and gastrointestinal tract ${ }^{1}$. Involvement of the nervous system by Mycoplasma can be focal or diffuse and diverse neurological manifestations are observed. Neurological manifestations include encephalitis, aseptic meningitis, cranial nerve palsy, psychosis, optic neuritis, Guillain-Barré syndrome, epilepsy, polyradiculitis, cerebellar ataxia, transverse myelitis and extrapyramidal symptoms ${ }^{4}$. Encephalitis is the most common form in pediatric patients and recent studies have suggested that Mycoplasma is responsible for 5 to $7 \%$ of cases of encephalitis among children ${ }^{5-8}$.

Involvement of the brainstem and cerebellum has been described during the course of inflammatory and infectious diseases such as multiple sclerosis, Bickerstaff's encephalitis, Behcet's disease, Lyme disease, progressive multifocal leukoencephalopathy, sarcoidosis, Whipple's disease, listeria rhombencephalitis, vasculitis due to systemic lupus erythematosus, and acute disseminated encephalomyelitis ${ }^{9}$, but has been rarely reported for infection with M. pneumoniae.

The present patient showed preliminary gastrointestinal and upper airway signs, however, these signs are not specific of M. pneumoniae infection and have also been described as other types of encephalitis such as those caused by enterovirus ${ }^{6}$.

Different mechanisms have been suggested to explain CNS involvement, including direct invasion of the CNS, immune-mediated reaction, vascular injury, hypersensitivity, and toxicity ${ }^{2}$. In the case of direct invasion of the CNS, M. pneumoniae is isolated from blood and cerebrospinal fluid, a fact suggesting that this microorganism crosses the blood-brain barrier. Recently, Mycoplas$m a$ was detected by highly sensitive PCR in cerebrospinal fluid. In these cases, respiratory symptoms seem to be less common. Mycoplasma pneumoniae produces a neurotoxin that causes brain tissue damage, inducing cerebral polyarteritis ${ }^{1}$. Immune-mediated aggression is characterized by the presence of autoantibodies in brain tissues, provoking a distant antigen-antibody reaction, for example in extrapulmonary tissues ${ }^{1}$. The deposition of immune complexes causes secondary vasculopathy. Immunosuppression induced by the microorganism and vessel thrombosis determined by a trend toward hypercoagulability are other mechanisms cited in the pathogenesis of the disease $^{1}$. Molecular alterations result in an immune-mediated reaction, producing an inflammatory state of cerebral vessels which, together with hypercoagulability, leads to thrombotic processes ${ }^{2}$. The production of neurotoxins by some Mycoplasma species contributes to neurological damage and to the production of autoantibodies in cerebrospinal fluid ${ }^{2}$.

The diagnosis of CNS involvement is difficult because 
M. pneumoniae is rarely detected in cerebrospinal fluid. Thus, the diagnosis is generally made based on indirect findings such as the detection of specific IgM and positive PCR of nasopharyngeal and throat swabs ${ }^{3,5,10,11}$. Furthermore, the high prevalence of M. pneumoniae infections, false-positive responses of IgM antibody and colonization of the respiratory tract in healthy subjects make the diagnosis even more difficult and question the true role of this microorganism in CNS infections ${ }^{11}$.

The diagnosis of CNS infection with Mycoplasma can be classified as confirmed or likely ${ }^{11}$. The diagnosis is confirmed in the case of a PCR-positive cerebrospinal fluid sample, and is likely in the case of serological evidence of acute infection (positive IgM test or occurrence of IgG in samples collected between the acute and convalescence phase) and/or PCR-positive respiratory samples. However, PCR identification of $M$. pneumoniae in cerebrospinal fluid is variable and, according to Christie et al. ${ }^{6}$ and Daxboeck $^{10}$, may occur in only $2 \%$ and $14 \%$ of cases, respectively. The lack of detection of M. pneumoniae by PCR or culture does not exclude this agent as a cause of encephalitis. Factors that may explain false-negative PCR results are a prolonged interval between the onset of symptoms and collection of cerebrospinal fluid and the fact that neurological manifestations might be due to physiopathological mechanisms other than direct invasion ${ }^{10}$.

Radiological alterations are preferentially detected by MRI, with an enhanced signal being more commonly observed in white or diffuse matter on T2-weighted imag$\mathrm{es}^{12}$. However, MRI might be normal in up to $49 \%$ of cases of encephalitis ${ }^{6}$.

Macrolides are the treatment of choice in the case of respiratory manifestations, and may or may not be effective to treat CNS manifestations ${ }^{3}$. The different physiopathological mechanisms of CNS disease and the limited penetration of antibiotics into the CNS are responsible for doubts regarding the effectiveness of the agents ${ }^{5}$. Corticosteroids and human immunoglobulin might be considered after the exclusion of other causes when a possible immunological mechanism is suspected ${ }^{2}$. Plasmapheresis might be beneficial in transverse myelitis and polyradiculitis ${ }^{13,14}$. The present case showed a favorable evolution even without the administration of corticosteroids and antibiotics, suggesting that the disease may present a benign and self-limited course in certain patients who do not require specific treatment.

Encephalitis is a rare and potentially severe disease and many etiologies remain undefined in both children and adolescents. In this respect, $M$. pneumoniae should be added to the differential diagnosis even in the case of patients without pneumonia who present involvement of the brainstem and cerebellum, especially in view of the existence of antibiotics that specifically act against this agent and may benefit certain patients.

\section{REFERENCES}

1. Guleria R, Nisar N, Chawla TC, Biswas NR. Mycoplasma pneumoniae and central nervous system complications: a rewiew. J Lab Clin Med 2005;146:56-63.

2. Tsiodras S, Kelesidis T, Kelesidis I, Stamboulis E, Giamarellou H. Central nervous system manifestations of Mycoplasma pneumoniae infections. J Infect 2005;51: 343-354.

3. Daxboeck F. Mycoplasma pneumoniae central nervous infections. Curr Opin Neurol 2006;19:374-378.

4. Bielsa-Martín S, Porcel-Pérez JM, Madroñero-Vuelta AB. Acute cerebellitis caused by Mycoplasma pneumoniae. Rev Neurol 2005;41:128.

5. Bitnun A, Ford-Jones E, Blaser S, Richardson S. Mycoplasma pneumoniae ecephalitis. Semin Pediatr Infect Dis 2003;14:96-107.

6. Christie LJ, Honarmand S, Talkington DF, et al. Pediatric encephalitis: what is the role of Mycoplasma pneumoniae? Pediatrics 2007;120:305-313.

7. Smith R, Eviatar L. Neurologic manifestations of Mycoplasma pneumoniae infections: diverse spectrum of diseases. A report of six cases and review of the literature. Clin Pediatr (Phila) 2000;39:195-201.

8. Arakawa $\mathrm{H}$, Yuhara $\mathrm{Y}$, Todokoro $\mathrm{M}$, et al. Immunoadsorption therapy for a child with Guillain-Barré syndrome subsequent to Mycoplasma infection: a case study. Brain Dev 2005;27:431-433.

9. Falini A, Kesavadas C, Pontesilli S, Rovaris M, Scotti G. Differential diagnosis of posterior fossa multiple sclerosis lesions: neuroradiological aspects. Neurol Sci 2001:22(Suppl 2):S79-S83.

10. Daxboeck F, Blacky A, Seidl R, Krause R, Assadian O. Diagnosis, treatment, and prognosis of Mycoplasma pneumoniae childhood encephalitis: systematic review of 58 cases. J Child Neurol 2004;19:865-871.

11. Domenech C, Leveque N, Lina B, Najioullah F, Floret D. Role of Mycoplasma pneumoniae in pediatric encephalitis. Eur J Clin Microbiol Infect Dis 2009; 28:91-94.

12. Pellegrini M, O'Brien TJ, Hoy J, Sedal L. Mycoplasma pneumoniae infection associated with an acute brainstem syndrome. Acta Neurlo Scand 1996;90:203-206.

13. Lin WC, Lee PI, Lu CY, et al. Mycoplasma pneumoniae encephalitis in childhood. J Microbiol Imunol Infec. 2002;35:173-178.

14. Tsiodras S, Kelesidis Th, Kelesidis I, Voumbourakis K, Giamarellou H. Mycoplasma pneumoniae-asociated myelitis: a comprehensive review. Eur J Neurol 2006,13:112-124. 